12

\title{
О границах применимости приближения Хольма-Кольрауша для определения нагрева сильноточных симметричных электрических контактов
}

\author{
() Д.И. Бегаль, М.А. Павлейно, О.М. Павлейно, М.С. Сафонов, А.А. Статуя
}

Санкт-Петербургский государственный университет, физический фракультет, 198504 Петергоф, Санкт-Петербург, Россия

e-mail: pavleyno@mail.ru

Поступило в Редакцию 8 июня 2018 г.

В окончательной редакции 25 декабря 2018 г.

Принято к публикации 11 февраля 2019 г.

На основе численного моделирования нагрева симметричных контактов протекающим током сделаны выводы о точности определения температуры по методу Хольма-Кольрауша при различных способах охлаждения контактов, в том числе при интенсивном воздушном и жидкостном охлаждении. Рассмотрен вопрос о возможности применения данного метода при импульсном нагреве контактов. Показано, как зависит точность определения температуры контактных площадок от выбора мест измерения температуры поверхности и разности потенциалов.

DOI: 10.21883/JTF.2019.07.47806.229-18

\section{Введение}

Электрические контакты входят в состав практически каждой цепи и выполняют функцию ее коммутации. Область их применения чрезвычайно широка - от систем микроэлектроники до электрических аппаратов высоковольтных линий электропередач. Уровни коммутируемых токов и рабочих напряжений в подобных устройствах отличаются более чем на десять порядков, что ведет к разнообразию их конструкций. Общим для них является то, что контакты представляют собой физически различные проводники, которые способны проводить электрический ток при их непосредственном соприкосновении путем приложения внешних сил.

Ток проходит не через всю поверхность соприкосновения проводников, а лишь через небольшие по площади контактные пятна. Это вызвано двумя основными причинами. Первая из них связана с наличием поверхностных пленок с низкой проводимостью. Вторая вызвана тем, что поверхность контактов не является идеально гладкой и ее реальный профиль характеризуется наличием неоднородностей, параметры которых зависят от технологии изготовления изделия и способа обработки поверхности.

Плотность тока в окрестности контактных пятен существенно больше, чем в удаленных от них областях. Это приводит к тому, что места непосредственного контактирования характеризуются наличием дополнительного сопротивления по сравнению с однородными участками электрической цепи, называемого контактным сопротивлением [1-3]. Его наличие приводит к дополнительному тепловыделению.

Вследствие этого области непосредственного контактирования являются, как правило, наиболее нагретыми участками цепи. Это может вызвать их перегрев свыше предельно допустимых значений, что отрицательно сказывается на работоспособности самих контактов и электрической цепи в целом.

Особенно остро проблема возможного перегрева проявляется для сильноточных контактов, входящих, например, в состав высоковольтных электрических аппаратов. Предельно допустимый уровень нагрева их токоведущих систем, содержащих электрические контакты, строго регламентирован как в режиме протекания номинальных токов, так и при протекании токов короткого замыкания $[4,5]$.

Определение температуры контактов является непростой задачей. Прямое ее измерение в окрестности контактных пятен не представляется возможным из-за малости их размеров и больших градиентов температуры в этих областях даже при стационарном нагреве. С этой точки зрения большой практический интерес представляют косвенные методы, которые позволяют связать температуру контактных пятен с величинами, которые доступны для измерения. К таким величинам относятся разность потенциалов на контактном переходе $U_{c}$, величина протекающего тока и значение температуры на внешней поверхности контактов.

Например, для случая стационарного нагрева при отсутствии пленок на контактирующих поверхностях можно оценить температуру контактных пятен по измеренному значению $U_{c}$, сравнивая его с известными табличными величинами напряжения размягчения $U_{c}^{s o f t}$ и напряжения плавления $U_{c}^{\text {melt }}$ для металлов. Это позволяет судить о том, достигается ли в контакте температура размягчения или температура плавления при заданном уровне протекающего тока. 


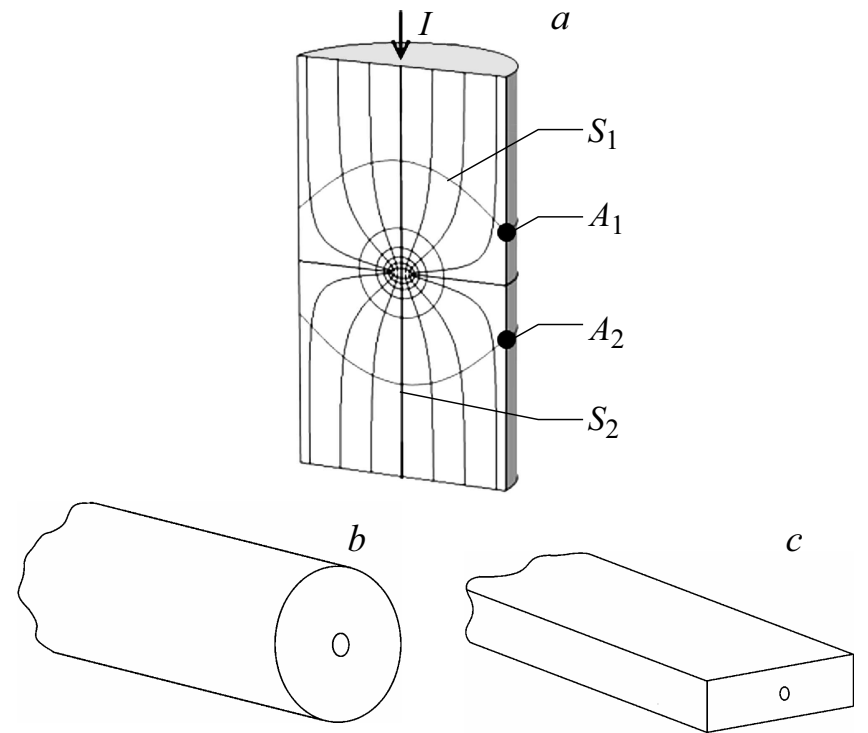

Рис. 1. Продольное сечение сильноточных симметричных контактов с линиями тока и эквипотенциалями $(a)$, торцевой контакт цилиндрических проводников $(b)$ и проводников прямоугольного сечения $(c)$ с обозначенным контактным пятном.

Более общим и наиболее распространенным в настоящее время косвенным способом оценки температуры контактных площадок является метод ХольмаКольрауша, предложенный в [1]. В этой работе рассмотрены симметричные контакты произвольной формы, соединенные одним полностью проводящим (нефрагментированным) контактным пятном (рис. 1,a).

Если предположить, что боковая поверхность адиабатична, то линии электрического тока и линии потока тепла совпадают, а любая эквипотенциальная поверхность является также изотермической, т.е. возбуждающиеся в контактах стационарные тепловое и электрическое поля имеют одинаковые конфигурации. Используя метод термоэлектрических аналогий, в [1] показано, что температуру контактного пятна $T_{c}$ можно связать с температурой $T_{12}$ двух симметричных изотермических поверхностей $S_{1}$ и $S_{2}$ и разностью потенциалов между ними $U_{12}$ (формула Хольма-Кольрауша):

$$
T_{c}=\sqrt{T_{12}^{2}+\frac{U_{12}^{2}}{4 L}},
$$

где $L$ - параметр Лоренца. При выводе (1) предполагается, что для материала контактов справедлив закон Видемана-Франца-Лоренца:

$$
\frac{\kappa}{\sigma}=L T,
$$

где $\kappa$ и $\sigma$ - теплопроводность и электропроводность материала.

Выражение (1) позволяет получить значение температуры контактного пятна путем измерения температуры в одной из точек $A_{1}$ или $A_{2}$ на поверхности контактов и разности потенциалов между ними.
Заметим, что модель с полностью проводящим контактным пятном достаточно хорошо описывает реальную ситуацию в случае сильноточных контактов. Для них характерны большие значения сил контактного нажатия, которые исчисляются десятками или даже сотнями ньютонов. В этом случае взаимодействующие поверхности подвержены значительным, в том числе пластическим, деформациям. Это снижает степень фрагментации контактных пятен, вызванную наличием шероховатости поверхности, и позволяет без заметного снижения точности результатов пренебречь этим эффектом [6].

Для реальных контактов и режимов прохождения тока через них условия, при которых получено выражение (1), могут быть заметно нарушены. Во-первых, всегда существует теплообмен контактов с окружающей средой. Выделяющееся в контактной окрестности тепло отводится не только вдоль проводников за счет теплопроводности, но и с их поверхности за счет механизмов конвекции и излучения. Во-вторых, протекающие через них токи могут быть нестационарны. Например, при коммутации нагрузок в электрических сетях или в результате возникновения коротких замыканий.

В этих случаях данный способ определения температуры контактных пятен становится приближенным.

\section{Проведение численных расчетов}

Исследуем влияние указанных факторов на точность определения температуры по методу Хольма-Кольрауша на примере торцевых контактов. Будем сравнивать приближенное значение температуры контактного пятна, рассчитанной из (1), с температурой, полученной в результате численного решения методом конечных элементов задачи о нагреве проводников заданной конфигурации протекающим стационарным или импульсным током.

Эти величины совпадают для симметричных контактов при задании адиабаты на их поверхности и начинают различаться по мере увеличения интенсивности теплообмена контактов с окружающей средой и уменьшении длительности импульса тока.

Численное моделирование проводится в программной системе ANSYS. Система уравнений, описывающая нагрев контактов протекающим током, имеет вид

$$
\begin{gathered}
\rho C \frac{\partial T}{\partial t}=-\nabla(\kappa \nabla T)=\frac{1}{\sigma} j^{2}, \\
\nabla^{2} \varphi=0, \\
\mathbf{E}=-\nabla \varphi \\
\mathbf{j}=\sigma \mathbf{E},
\end{gathered}
$$

где $T$ - температура, $\mathbf{j}$ - плотность электрического тока, $\varphi$ - электрический потенциал, $\mathbf{E}-$ напряженность электрического поля. При решении задачи учитывается зависимость теплопроводности $\kappa$, теплоемкости $C$, 
плотности $\rho$ и электропроводности $\sigma$ материала от температуры.

Приведенная система уравнений записана в приближении отсутствия влияния вихревых токов на нагрев контактов, что допустимо, если толщина скин-слоя гораздо больше радиуса контактного пятна. Для рассматриваемых в настоящей работе сильноточных медных контактов с характерным размером контактного пятна $1 \mathrm{~mm}$ такое приближение будет справедливо при длительности токовых воздействий более $1 \mathrm{~ms}$.

Теплообмен с окружающей средой учитывается путем задания на боковой поверхности контактов коэффициента серости (0.9 в последующих расчетах) и коэффициента конвекции. Варьируя коэффициент конвекции, мы можем моделировать разные механизмы охлаждения. В работе [7] приведены типичные значения $k$ для сильноточных контактов: $k<10 \mathrm{~W} /\left(\mathrm{m}^{2} \mathrm{~K}\right)$ для воздушного охлаждения за счет естественной конвекции, $k=10-100 \mathrm{~W} /\left(\mathrm{m}^{2} \mathrm{~K}\right)$ - принудительное воздушное охлаждение, $k=200-600 \mathrm{~W} /\left(\mathrm{m}^{2} \mathrm{~K}\right)-$ автономное жидкостное охлаждение, $k=1000-3000 \mathrm{~W} /\left(\mathrm{m}^{2} \mathrm{~K}\right)$ принудительное жидкостное охлаждение.

В расчетах будут использованы модели торцевых соединений двух медных протяженных проводников (рис. $1, b, c$ ) круглого (радиус $r$ ) и прямоугольного (длины сторон $b$ и $c$ ) сечений, соединенных круглым проводящим контактным пятном радиуса $a$. Контактное пятно может быть смещено относительно оси симметрии контактов на расстояние $d$.

При построении конечноэлементной модели учитывается наличие больших градиентов температуры вблизи контактного пятна, особенно при импульсном нагреве. Размер элементов в контактной окрестности выбран на два порядка меньше радиуса контактного пятна, по мере удаления от этой области он увеличивается до значения $a$.

\section{Стационарный нагрев сильноточных контактов}

Рассмотрим стационарный режим нагрева симметричных контактов. Будем оценивать точность расчета температуры по методу Хольма-Кольрауша, зафиксировав величину перегрева пятна относительно температуры окружающей среды в $100^{\circ}$. Выбор этого значения продиктован тем, что допустимые перегревы сильноточных контактов при их стационарном нагреве номинальными токами не превосходят $100^{\circ}$ [4,5]. Таким образом, при выбранных геометрических параметрах контактов и выбранной величине коэффициента конвекции величина тока подбирается такой, чтобы при его протекании был обеспечен указанный перегрев.

В результате численного решения задачи для заданных геометрических параметров электродов, выбранном размере и положении контактного пятна, выбранном значении коэффициента конвекции и коэффициента серости рассчитываются пространственные распределения электрического и теплового поля контактов: потенциала и напряженности электрического поля, температуры, в том числе на поверхности контактного пятна, и плотности теплового потока.

При расчете температуры контактного пятна по методу Хольма-Кольрауша нужно выбрать расположение точек $A_{1}$ и $A_{2}$ для определения значения температуры $T_{12}$ и разности потенциалов $U_{12}$, которые будут подставлены в (1). Введем параметр $s$, отвечающий за их выбор: это расстояние вдоль оси симметрии контактов от поверхности контактного пятна до соответствующей адиабаты (изотермы) $S_{1}$ или $S_{2}$.

При моделировании нагрева контактов величина параметра $s$ может варьировать в произвольных пределах. В реальности минимальное значение $s_{\min }$ ограничено тем, насколько близко к пятну могут быть расположены датчики температуры и зонды для измерения разности потенциалов. Для разных конструкций контактов удаленность точек возможного измерения указанных величин от контактного пятна может сильно различаться, но в подавляющем большинстве случаев $s_{\min } \gg a$.

Для случая адиабатичной поверхности температура, рассчитанная по (1), совпадает с результатами численного расчета этой температуры при любом значении параметра $s$. При наличии теплообмена с окружающей средой погрешность определения температуры по (1) зависит от места положения точек, где измеряется температура и разность потенциалов, а также от метода охлаждения контактов.

Точность расчета температуры контактных пятен методом Хольма-Кольрауша будем оценивать величиной $\Delta T=\frac{T_{H K}-T_{M}}{T_{M}} 100 \%$, где $T_{H K}-$ значение температуры контактного пятна, полученное из (1), $T_{M}$ - температура, полученная компьютерным моделированием в ANSYS.

Рассмотрим цилиндрические контакты, радиус которых на порядок больше радиуса контактного пятна $r=10 a$, а само пятно расположено на оси симметрии. Назовем такую конфигурацию контактов исходной. На рис. 2, $a$ представлено семейство зависимостей величины $\Delta T$ от места расположения измерительных точек (параметр $s / a$ ) для широкого диапазона значений коэффициента конвекции (разных способах охлаждения контактов).

Для таких контактов метод Хольма-Кольрауша дает достаточно точную (с ошибкой менее 10\%) оценку температуры контактного пятна для воздушного охлаждения контактов. Причем измерения температуры и разности потенциалов можно проводить на значительном удалении от контактной поверхности, вплоть до $100 \mathrm{a}$. Если охлаждение более интенсивное, например жидкостное, то места расположения точек измерения ограничены гораздо более жесткими условиями. 

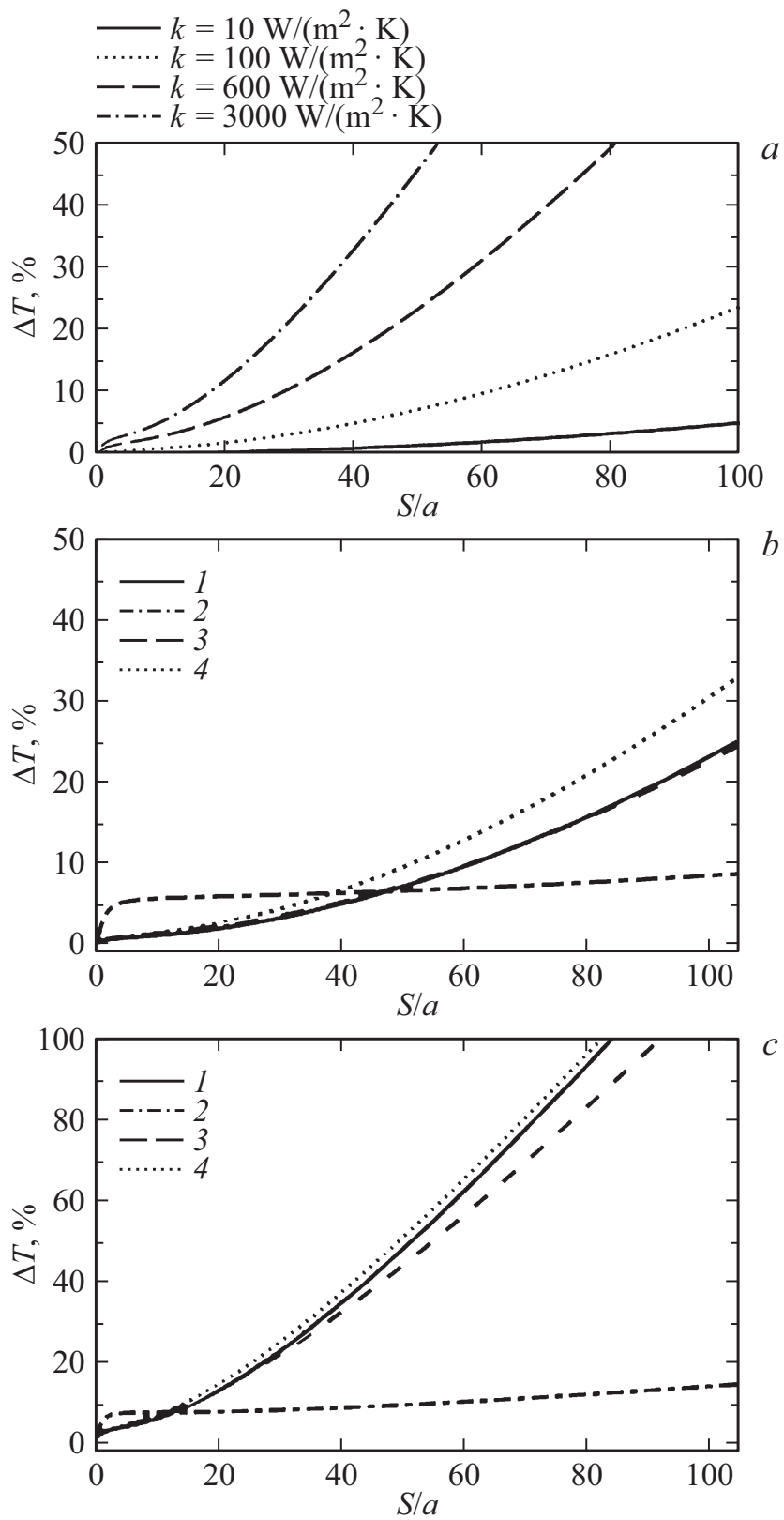

Рис. 2. Точность определения температуры контактных пятен по методу Хольма-Кольрауша при стационарном нагреве.

Определим, насколько изменится точность определения температуры по (1) при значительном изменении конфигурации контактов относительно исходной. Расчеты будем проводить для интенсивного воздушного охлаждения при $k=100 \mathrm{~W} /\left(\mathrm{m}^{2} \mathrm{~K}\right)$ и для интенсивного жидкостного охлаждения при $k=3000 \mathrm{~W} /\left(\mathrm{m}^{2} \mathrm{~K}\right)$.

Для этих двух случаев на рис. 2, $b, c$ приведены семейства кривых $\Delta T(s / a)$ для контактов исходной конфигурации (кривые 1); для контактов той же конфигурации с увеличенным на порядок отношением площади проводника к площади контактного пятна (кривые 2); для пятна, смещенного на расстояние $d=r-2 a$, т.е. расположенного практически вплотную к боковой гра- нице цилиндрических контактов (кривые 3); для проводников прямоугольного сечения с соотношением сторон $c / b=5$ той же площади, что и в исходном случае (кривые 4).

Для контактов с воздушным охлаждением проведенное существенное изменение размеров и формы сечения проводников и изменения расположения контактного пятна в пределах плоскости их соприкосновения не оказали заметного влияния на точность метода ХольмаКольрауша. Подобные изменения заметно влияют на величину контактного сопротивления [8] и, следовательно, на температуру контактного пятна при фиксированном токе, но не на точность ее расчета по (1).

Для контактов с жидкостным охлаждением изменения погрешности метода более заметны. Но путем правильного выбора места расположения точек измерения разности потенциалов и температуры можно обеспечить требуемую точность оценки температуры контактных пятен.

\section{Импульсный нагрев сильноточных контактов}

Если говорить о сильноточных контактах, использующихся в устройствах коммутации высоковольтных электрических сетей, то практический интерес вызывают импульсные режимы токовых воздействий, составляющие единицы секунд и десятки миллисекунд. Первый режим соответствует прохождению через контакты токов короткого замыкания. Согласно нормативным документам [9], коммутационные аппараты должны выдерживать протекание таких токов в течение 1-4s без потери работоспособности. Второй режим соответствует прохождению ударных аварийных токов, длительность которых сравнима с периодом токов промышленной частоты [9].

В этих режимах характерные значения протекающего тока составляют единицы и десятки $\mathrm{kA}$, что сужает возможности проведения экспериментального исследования нагрева контактов, так как требует наличия специального оборудования - генераторов тока указанного уровня, имеющихся в основном в специализированных сертификационных центрах.

В отличие от стационарного нагрева, при нагреве секундными или миллисекундными импульсами допускаются существенно большие перегревы контактных поверхностей. Наиболее значимым негативным явлением при кратковременном нагреве является сваривание контактов. Оно наступает при температурах, гораздо меньших температуры плавления, за счет диффузионных механизмов. Так, например, для медных контактов при протекании ударных токов сварка наблюдается уже при $700 \mathrm{~K}$ [10]. Кратковременный нагрев медных контактов до температуры $500 \mathrm{~K}$ в большинстве случаев можно считать безопасным. Исходя из этого, при выполнении 

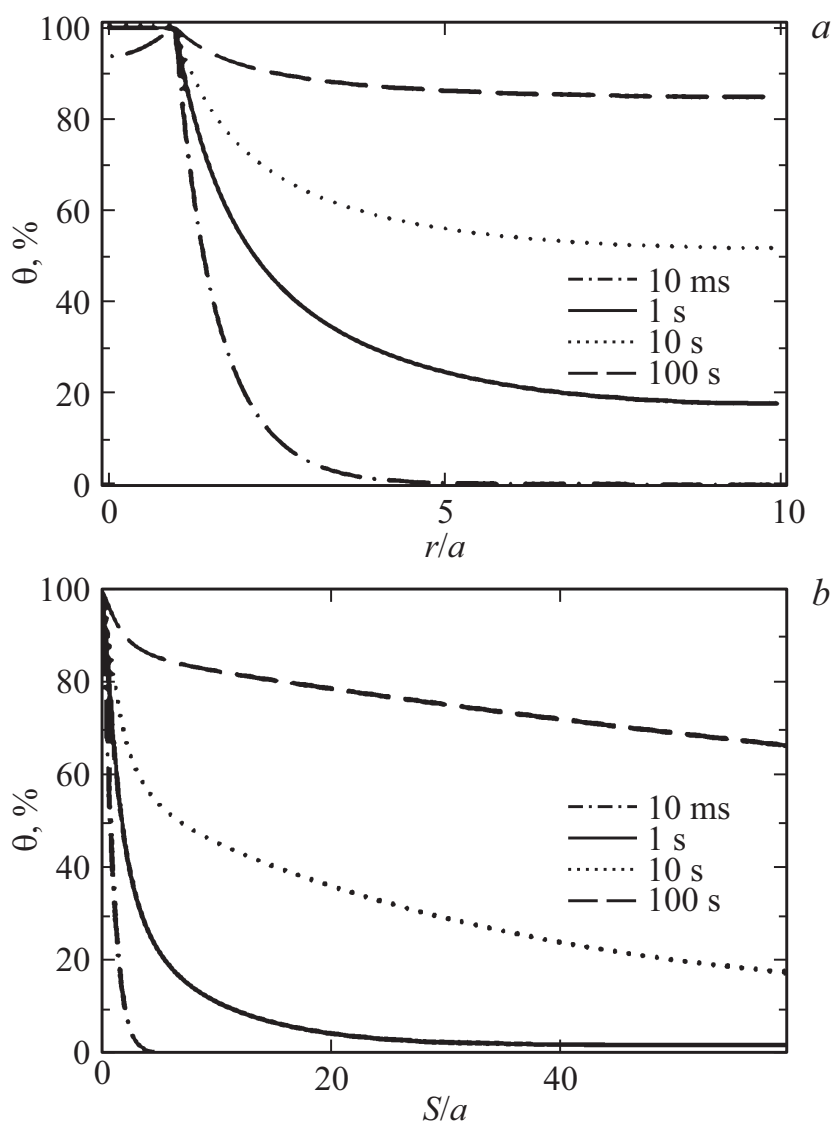

Рис. 3. Распределение температуры в окрестности контактного пятна: вдоль поверхности соприкосновения проводников $(a)$ и вдоль оси симметрии $(b)$.

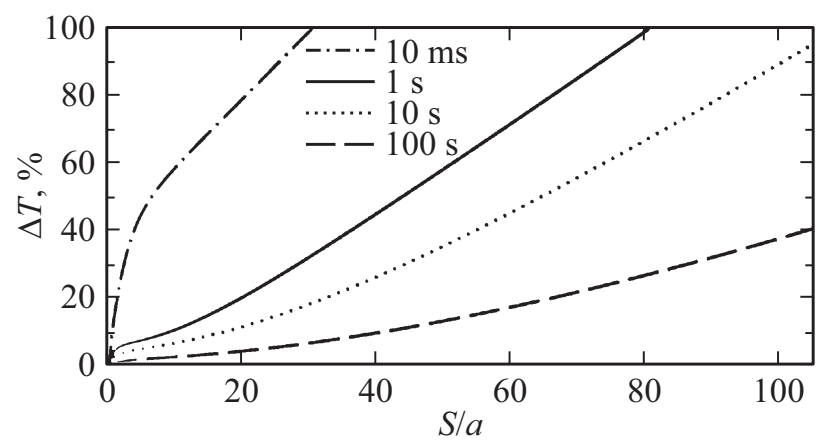

Pис. 4. Точность определения температуры контактных пятен по методу Хольма-Кольрауша при нестационарном нагреве.

численных расчетов будем подбирать величину протекающего тока для каждого значения его длительности так, чтобы максимальная температура контактного пятна была равна $500 \mathrm{~K}$.

При указанных длительностях нагрева теплообмен с окружающей средой практически не оказывает влияния на температуру самих контактов и всей токоведущей системы в целом. Поэтому при моделировании будем задавать условие адиабаты на боковой поверхности контактов.
Причиной возникновения погрешности расчета температуры методом Хольма-Кольрауша является нестационарность теплового поля. Чем меньше длительность нагрева, тем больше различие в структуре электрического и теплового полей, и больше погрешность определения температуры по (1). Сделаем количественные оценки погрешности, рассмотрев исходную конфигурацию электродов при изменении длительности от $10 \mathrm{~ms}$ до $100 \mathrm{~s}$.

Проанализируем распределение температуры $\theta=\frac{T-T_{0}}{T_{\max }-T_{0}} 100 \%$ в окрестности контактного пятна, где $T_{\max }$ - максимальная температура, $T_{0}$ - начальная температура. На рис. 3 приведены графики изменения температуры от расстояния от центра контактного пятна вдоль оси $O X$, проходящей по торцевой поверхности, и $O Y$, совпадающей с осью симметрии.

При длительности нагрева более $1 \mathrm{~s}$ температура в пределах контактного пятна практически постоянна. При меньших длительностях контактное пятно нагрето неравномерно, и в этом случае под температурой пятна будем понимать ее максимальное значение, достигаемое на краю пятна.

Результаты сопоставления расчета температуры по (1) и на основе компьютерного моделирования приведены на рис. 4. Представленные зависимости позволяют заключить, что оценка нагрева контактов по (1) при длительностях нагрева менее $10 \mathrm{~ms}$ обладает погрешностью более $50 \%$ даже в том случае, когда измерительные зонды расположены в непосредственной близости от поверхности соприкосновения контактов. При длительностях нагрева более $1 \mathrm{~s}$ можно указать такие места расположения зондов, где погрешность не будет превосходить $10-15 \%$, что с практической точки зрения может оказаться приемлемым для проведения оценок.

\section{Выводы}

Проведенные расчеты позволяют сделать практически значимые выводы о том, как связана погрешность вычисления температуры контактных пятен по методу Хольма-Кольрауша с интенсивностью теплообмена с окружающей средой и местом расположения датчиков температуры и разности потенциалов, а также с длительностью протекающего тока.

Метод Хольма-Кольрауша является эффективным способом оценки температуры контактных пятен для стационарного режима нагрева сильноточных контактов как с воздушным, так и с жидкостным охлаждением. При соответствующем выборе мест расположения зондов для измерения температуры поверхности контактов и разности потенциалов погрешность метода не превышает $10 \%$.

Данный метод может быть использован также для оценки температуры контактных пятен при импульсном нагреве, если длительность протекания тока более $1 \mathrm{~s}$, что соответствует режиму протекания токов термической стойкости через сильноточные контакты. 
При использовании метода Хольма-Кольрауша для режима протекания ударных токов короткого замыкания погрешность определения температуры оказывается неприемлемо большой.

\section{Список литературы}

[1] Хольм Р. Электрические контакты. М.: ИЛ, 1961.

[2] Braunovic M., Konchits V.V., Myshkin N.K. Electrical Contacts. Fundamentals, Applications and Technology. NY: Taylor \& Francis Group, 2006. 495 p.

[3] Slade P.G. Electrical Contacts: Principles and Applications. NY.: CRC Press, 2014.

[4] ГОСТ 8024-90 Аппараты и электротехнические устройства переменного тока на напряжение свыше 1000 В. Нормы нагрева при продолжительном режиме работы и методы испытаний. М.: Изд-во стандартов, 1990.

[5] ГОСТ Р 52565-2006 Выключатели переменного тока на напряжения от 3 до 750 кВ. Общие технические условия. М.: Стандартинформ, 2007.

[6] Павлейно О.М. // Современные проблемы науки и образования. 2014. № 2. С. 665.

[7] Киладзе И.Е., Павлейно М.А., Павлейно О.М., Павлов В.A. Оценка погрешности определения температуры электрических контактов в приближении ХольмаКольрауша. // Сб. докл. Х Междунар. науч. конф. „Современные проблемы электрофизики и электрогидродинамики жидкостей“ СПб. 2012. С. 220-222.

[8] Pavleino O.M., Pavlov V.A., Pavleino M.A. // Surf. Engineer. Appl. Electrochem. 2010. Vol. 46. N 5. P. 440-446.

[9] ГОСТ Р 52736-2007 Короткие замыкания в электроустановках. Методы расчета электродинамического и термического действия тока короткого замыкания. М.: Стандартинформ, 2007.

[10] Автореф. канд. дис. Павлейно О.М. Физические особенности нагрева сильноточных электрических контактов. 01.04.13. Институт электрофизики и электроэнергетики РАН. СПб., 2015. 148 c. 